\title{
Uma abordagem de modelagem de conteúdo educacional móvel baseada na Engenharia Dirigida por Modelo
}

\author{
Marcus Vinicius R. Carvalho ${ }^{1}$, Ellen Francine Barbosa ${ }^{1}$ \\ ${ }^{1}$ Instituto de Ciências Matemáticas e de Computação (ICMC) \\ Universidade de São Paulo, Caixa Postal 668 - 13560-970 \\ São Carlos - SP - Brasil \\ mvcarvalhopieusp.br, francine@usp.br
}

\begin{abstract}
The increased use of mobile devices in various sectors has contributed to modify social behavior. In the educational context, mobile devices have significantly modified the teaching and learning process. However, challenges such as screen size and structure of educational content are still being faced by teachers and application developers. In order to minimize educational content modeling efforts for mobile devices, this paper presents a proposal for modeling mobile educational content based on the AIM-CID approach and Model Driven Engineering (MDE). By way of illustration, the proposed modeling is applied and discussed in the field of object-oriented programming teaching.
\end{abstract}

Resumo. O aumento do uso de dispositivos móveis nos mais variados setores tem contribuído para modificar comportamentos sociais. No contexto educacional, dispositivos móveis têm modificado, de forma significativa, o processo de ensino e aprendizagem. No entanto, desafios como tamanho de tela e estrutura do conteúdo educacional ainda são enfrentados por professores e desenvolvedores de aplicativos. No sentido de minimizar os esforços de modelagem de conteúdo educacional para dispositivos móveis, este artigo apresenta uma proposta de modelagem de conteúdo educacional móvel, tendo como base a abordagem AIM-CID e a Engenharia Dirigida por Modelos (MDE). A título de ilustração, a modelagem proposta é aplicada e discutida no domínio de ensino de programação orientada a objetos.

\section{Introdução}

Segundo pesquisa IBGE-PNAD 2014 [IBGE 2018], no Brasil o uso de dispositivos móveis para acessar a Internet já havia superado o uso de computadores. Outra pesquisa, realizada pela GSMA Itelligence em 2016 [Association 2017], apontou que 65\% da população mundial possuía uma assinatura móvel. Esse cenário é oportuno para introduzir dispositivos móveis no processo de ensino e aprendizagem. Recursos como mobilidade, visualização de vídeos e áudio tornam os dispositivos móveis uma ferramenta educacional atraente ao aprendiz.

No entanto, desafios tecnológicos, sociais e metodológicos precisam ser enfrentados por professores e desenvolvedores de aplicativos móveis [Prieto et al. 2013][Diwanji et al. 2014][Navarro et al. 2016]. Em dispositivos móveis o conteúdo educacional precisa ser adequado a telas reduzidas. Além disso, desafios como o grau de instrução de usuários, distrações derivadas do contexto multimídia 
VII Congresso Brasileiro de Informática na Educação (CBIE 2018)

Anais do XXIX Simpósio Brasileiro de Informática na Educação (SBIE 2018)

e móvel e estrutura de conteúdo compatível com dispositivos móveis precisam ser minimizados[Diwanji et al. 2014].

Considerando o contexto da estruturação de conteúdo educacional e da diversidade de plataformas de consumo, este artigo propõe a modelagem de conteúdo educacional móvel baseada na abordagem AIM-CID (Abordagem de Modelagem Integrada, Conceitual, Instrucional e Didática)[Barbosa and Maldonado 2011] e na Engenharia Dirigida por Modelos (MDE)[Whittle et al. 2014]. Basicamente, a proposta faz uso da AIMCID para estruturar o conteúdo educacional e utiliza a MDE para minimizar o esforço de geração do conteúdo para uma ou mais plataformas tecnológicas de consumo.

A AIM-CID propõe uma modelagem de conteúdo educacional guiada pela construção de modelos que expressam os conceitos centrais de um domínio (Modelo Conceitual), bem como, elementos (exercícios, procedimentos, avaliação entre outros) que complementam tais conceitos (Modelo Instrucional). A AIM-CID modela conteúdo educacional desconsiderando aspectos tecnológicos, portanto, a fim de orientar professores/autores a estruturar conteúdo para dispositivos móveis de forma adequada, um conjunto de diretrizes de design de UI foi definido com base em levantamento da literatura e, também com base em propostas de fabricantes. A adoção da AIM-CID para estruturar o conteúdo educacional e da MDE para modelar e gerar conteúdo educacional móvel de forma semi-automática pode contribuir para minimizar o esforço de produção de conteúdo educacional.

Este artigo está organizado da seguinte forma. A Seção 2 apresenta uma síntese de trabalhos relacionados. A Seção 3 detalha o design de UI identificados para dispositivos móveis. A Seção 4 discute o processo de modelagem e geração de conteúdo educacional. A Seção 5 aplica a modelagem proposta no domínio de ensino de programação orientada a objeto. E, por fim, a Seção 6 sumariza as conclusões deste trabalho e as principais perspectivas para a continuidade da pesquisa.

\section{Trabalhos Relacionados}

Souza et al. [DE SOUZA 2012] propõem uma estratégia de customização guiada para produção de Learning Object (OA). O termo guiado está relacionado com a possibilidade de conduzir o professor na adaptação de Recursos Educacionais Digitais (RED). Um processo de produção denominado Customizable LeARning Objects Model-driven (CLAssRoOM) foi desenvolvido e consiste de três etapas: i) Descrição dos Requisitos, ii) Desenvolvimento de Componentes Gráficos ou simplesmente Modelagem; e iii) Implementação de Ações. A especificação da descrição dos requisitos são utilizadas para definição da arquitetura de OA Customizáveis (OAC), por meio do uso de uma Linguagem Específica de Domínio (DSL). A arquitetura produzida pela DSL serve de base para gerar, aplicando regras de transformações, o roteiro de atividades a ser utilizado pela equipe de projeto. Os templates de implementação são utilizados pela equipe de programação para adequações nos códigos gerados e obtenção do OA.

Minovic et al. [Minović et al. 2009] propõem uma modelagem de OAs para jogos educacionais apoiado na abordagem dirigida por modelo. Foram definidos metamodelos para descrever as seguintes características de jogos educacionais: conceitos básicos, domínio do conhecimento e OAs. A abordagem é baseada na Unified Modeling Language (UML) e no XML. Um metamodelo de conceitos básicos de jogos educacionais 
VII Congresso Brasileiro de Informática na Educação (CBIE 2018)

Anais do XXIX Simpósio Brasileiro de Informática na Educação (SBIE 2018)

foi definido e tem em sua composição mecanismos para apresentar o conhecimento, para gerenciar a interação entre jogador e game e controlar o progresso do aprendiz no game. A proposta tem como foco o conteúdo educacional para jogos.

A abordagem apresentada neste trabalho, difere das demais por propor uma produção de conteúdo educacional mais versátil. Isto é, ao dividir a modelagem em uma visão de estruturação de conteúdo livre de aspectos tecnológicos e uma outra visão da tecnologia de consumo do conteúdo, as responsabilidades de professor/autor e equipe de desenvolvimento são melhor atribuídas. Além disso, as sugestões de design de UI contribuem para orientar a equipe de produção na estruturação adequada do material didático para dispositivos móveis.

\section{Design de UI para conteúdo educacional móvel}

A tarefa de produzir conteúdo educacional demanda tempo e esforços de profissionais de Tecnologia da Informação (TI) e educação [Conde et al. 2012][Barbosa et al. 2013]. Além disso, cada plataforma tecnológica apresenta suas restrições e requisitos diferentes na produção do conteúdo educacional. Quando o conteúdo educacional é consumido por dispositivos móveis, restrições como limite de espaço de armazenamento, tamanho de tela, tamanho de textos precisam ser considerados pela equipe de produção de conteúdo [Joorabchi et al. 2013]. Algumas dessas restrições foram enfrentadas por desenvolvedores de software para domínios não educacionais com design de interfaces de usuário (UI). Portanto, as diretrizes de design de UI para desenvolvimento de aplicações móveis podem contribuir para auxiliar a modelagem de conteúdo educacional móvel.

Nesse sentido, esse trabalho levantou diretrizes propostas na literatura e pelos fabricantes para construção de UI para dispositivos móveis[Chittaro 2010] [Biard et al. 2017] [Apple 2018] [Google 2018]. Foram consideradas as diretrizes relacionadas com a apresentação de textos, imagens e vídeos. Além disso, as diretrizes foram classificadas em didáticas [D], em que professor/autores são responsáveis em aplicá-las, e em técnicas[T], que merecem atenção da equipe de desenvolvimento. A seguir as diretrizes levantadas são apresentadas.

\subsection{Imagem}

Fotos e ilustrações caracterizam conceitos de uma forma mais preditiva para os usuários de aplicações móveis[Google 2018]. Contudo, requisitos de produção de imagem precisam ser observados para atingir expressividade. Nesse sentido, as seguintes diretrizes de design UI foram identificadas: i) Atenção a estética e relevância: utilize imagens que tornem a compreensão fácil e imediata[Apple 2018][D]. ii) Faça uso de ilustrações e fotografia: fotografia são melhores aproveitadas para expressar entidade e história especificas, enquanto ilustrações representam melhor conceitos e metáforas [Apple 2018] [D]. iii) Mantenha um foco icônico na imagem: uma imagem com um foco claro expressa um conceito de forma imediata [Apple 2018] [D/T]. iv) Produza imagens com uma construção narrativa: imagens que contam uma história são mais informativas e compreensíveis [Google 2018][D]. v) Atenção a dimensões e resolução da imagem: as dimensões devem ser adequadas ao tamanho de tela e plataformas [Google 2018] [Apple 2018]. Além disso, a resolução deve permitir a ação de zoom sem a degradação da imagem $[\mathrm{D} / \mathrm{T}]$. 
VII Congresso Brasileiro de Informática na Educação (CBIE 2018)

Anais do XXIX Simpósio Brasileiro de Informática na Educação (SBIE 2018)

\subsection{Texto}

Ao produzir conteúdo textual, professor/autor precisam dedicar atenção a questões como a carga cognitiva de informação e entrada de dados[Chittaro 2010]. Como recomendações didáticas e técnicas para produção de conteúdo textual destacam-se: i) Tamanho da linha de texto. Segundo diretrizes de fabricantes de SO para dispositivos móveis, 60 caracteres por linha garante uma boa legibilidade do texto [Google 2018]. Porém, deve-se ter o cuidado com linhas muito curtas, pois prejudicam a legibilidade [Google 2018] [D/T]. ii) Escolha de fonte e cores. A variação de tamanho de fonte e cores é uma forma de enfatizar itens importantes no aplicativo [Apple 2018] [D/T]. iii) Ajuste de quebra de linha. Na quebra de linha evite deixar grandes lacunas, preposições ou palavras curtas no final de linha [Google 2018] [T]. iv) Utilize sensores dos dispositivos móveis. Os recursos sensoriais dos dispositivos móveis (captura de áudio, vídeo, GPS, câmera) podem auxiliar a entrada de dados [T].

\subsection{Vídeo}

Segundo o site Consumer Barometer Google, em 2014, pelo menos uma vez por semana, $22 \%$ dos usuários conectados à Internet utilizavam smartphone para acessar vídeos online no Reino Unido. Essa mesma pesquisa levantou que $54 \%$ das pessoas conectadas à Internet no Brasil acessavam vídeos on-line por meio de smartphone diariamente. Esses dados demonstram o potencial do uso de vídeo como ferramenta de aprendizagem. Contudo, a produção de vídeos para ambientes móveis requer atenção. Nesse sentido, a seguir são apresentadas algumas das diretrizes levantadas para produção de vídeo: i) Tempo do vídeo. Conforme pesquisa Consumer Barometer Google, os usuários sentem-se mais confortáveis visualizando vídeos com duração em torno de 5 minutos [D]. ii) Segmente o conteúdo. A divisão do conteúdo em porções menores melhora a retenção do conhecimento [Diwanji et al. 2014] [Biard et al. 2017] [Mayer 2017]. iii) Conteúdo relevante ao curso. Vídeos que apresentam conteúdo diretamente relacionados ao conteúdo do programa do curso são mais visualizados [Diwanji et al. 2014] [D]. iv) Qualidade de edição do vídeo. Vídeos com uma aparência profissional, com recursos e visual de qualidade são mais envolventes [Diwanji et al. 2014] [D/T]. v) Mantenha um discurso envolvente. Produza vídeo com diálogo mais leve e bem humorado, além disso, mantenha uma velocidade do discurso que não cause monotonia no aprendiz [Diwanji et al. 2014][D].

\section{Modelagem de conteúdo educacional móvel}

A modelagem de conteúdo educacional móvel proposta tem sua origem na AIM-CID. A AIM-CID propõe modelos que estruturam o conteúdo educacional desvinculada da modalidade de ensino e aprendizagem alvo (Web, móvel, tradicional). Além dos modelos Conceitual, Instrucional e Didático presentes na AIM-CID, foram propostos os modelos Mobile Learning e Modelo XMLAndroid, que modelam a plataforma tecnológica alvo.

Ademais, a AIM-CID permite a professores/autores adaptar o conteúdo modelado ao objetivo da aprendizagem. Essa adaptação é obtida a partir de três visões do domínio do conhecimento presentes na AIM-CID. A primeira visão apresenta os conceitos que serão estudados pelo aprendiz (Modelo Conceitual). A segunda elucida os conceitos modelados (Modelo Instrucional) e, por fim, tem-se a visão de navegabilidade dos conceitos (Modelo Didático). A seguir, os modelos que compõe a modelagem de conteúdo educacional móvel são descritos. 
VII Congresso Brasileiro de Informática na Educação (CBIE 2018)

Anais do XXIX Simpósio Brasileiro de Informática na Educação (SBIE 2018)

\section{Modelo conceitual}

O Modelo Conceitual é uma representação de alto nível dos conceitos relevantes do domínio do conhecimento[Barbosa et al. 2013]. Em suma, elementos do domínio do conhecimento precisam ser identificados, bem como os relacionamentos existentes entre eles. No Modelo Conceitual, os relacionamentos são classificados como estruturais e específicos do domínio. Relacionamentos estruturais representam relacionamentos genéricos presentes em qualquer domínio, sendo categorizados como associação de classificação type-of ou de composição part-of. Já os relacionamentos específicos do domínio representam relações que ocorrem em um determinado domínio .

Para atender aos fundamentos da MDE foi proposto um metamodelo para o Modelo Conceitual. O Metamodelo Conceitual é composto pelas seguintes classes: i) $C K$ nowledgeDomain, que representa o domínio do conhecimento. A classe CKnowledgeDomain é composta por um conjunto de módulos(CModule) ou conceitos (CConcept). ii) CModule, que possibilita a segmentação do conteúdo em módulos facilitando o planejamento do ensino. iii) CConcept, classe responsável em representar os conceitos relevantes de um domínio. iv) CReference, classe responsável em representar os relacionamentos entre os conceitos do domínio.

Em linhas gerais, a construção do Modelo Conceitual tem início com a segmentação do conteúdo em módulos de aprendizado. Para cada módulo, o professor/autor identifica os conceitos relevantes e atribui os inter-relacionamentos. Esse processo de identificação de conceitos e relacionamentos prossegue para todos os módulos do domínio do conhecimento.

\section{Modelo instrucional}

O objetivo do Modelo Instrucional é esclarecer, por meio de informações adicionais, os conceitos representados no Modelo Conceitual. Para elucidar os conceitos o professor/autor pode fazer uso de textos, imagens, exercícios, vídeos. Convém ressaltar que a construção do Modelo Instrucional para conteúdo educacional móvel segue as sugestões descritas na Seção 3.

A construção do Modelo Instrucional envolve as etapas de refinamento do Modelo Conceitual, definição de elementos instrucionais e caracterização de mídias. Na etapa de refinamento do Modelo Conceitual são definidos os itens de informação que representam elementos que explicam os conceitos modelados. Os itens de informação são especificados por meio de conceitos, fatos, procedimentos e princípios [Merrill 1983].

Enquanto os itens de informação apresentam a fundamentação teórica ao aprendiz, os elementos instrucionais têm o objetivo de reforçar conceitos e avaliar a retenção da aprendizagem. Com esse fim, elementos instrucionais são categorizados como: i) Elementos Explanatórios, que são informações que explicam um dado item de informação (exemplos, dicas, sugestões, estudo); ii) Elementos Exploratórios, que são exercícios, simulações, ferramentas; iii) Elementos de Avaliação, responsáveis por aferir o aprendizado.

O Modelo Instrucional é um modelo flexível, pois fornece ao professor/autor liberdade de criar modelos que atendem audiência diversificada e grau de aprofundamento de conteúdos diverso. Ou seja, o professor/autor pode construir modelos instrucionais para um público específico ou objetivos de ensino diferentes. 
VII Congresso Brasileiro de Informática na Educação (CBIE 2018)

Anais do XXIX Simpósio Brasileiro de Informática na Educação (SBIE 2018)

Seguindo a abordagem MDE, um Metamodelo Instrucional foi proposto para instanciar Modelos Instrucionais da AIM-CID. O Modelo Instrucional é obtido pela aplicação de regras de transformação de modelos ao Modelo Conceitual. O Modelo Instrucional instanciado é complementado com itens de informações e elementos instrucionais. As principais classes do metamodelo instrucional são apresentadas a seguir: i) IknowledgeDomain e IConceptual: as classes IknowledgeDomain e IConceptual são instanciadas, por regras de transformação de modelos, a partir do Modelo Conceitual representando o conjunto de conceitos relevantes do domínio modelado. ii) InformationItem: a classe InformationItem generaliza as classes Principle, Concept, Procedure ou Fact, que descrevem um conceito específico. iii) InstructionalElement: um (InformationItem) pode ser complementado pelas classes Example (ExplanatoryElement) ou um Exercise (ExploratoryElement) ou uma Avaluation (EvalueativeElement), que são elementos InstructionalElement que reforçam a compreensão de um conceito específico.

Os metamodelos Conceitual e Instrucional representam uma visão do conteúdo educacional desvinculada da plataforma tecnológica alvo. Portanto cada plataforma tecnologia alvo precisa da construção de metamodelos específicos.

\section{Metamodelo Mobile}

Como visto, umas das visões proposta na modelagem de conteúdo educacional móvel modela a plataforma alvo de consumo do conteúdo. Neste trabalho, o conteúdo educacional destina-se à plataforma Android. Vale ressaltar que o produto final da modelagem não é o aplicativo móvel, mas sim o "conteúdo educacional móvel". Com esse intuito, um Metamodelo Mobile foi proposto para representar as caraterísticas tecnológicas de dispositivos móveis. O Metamodelo Mobile é composto pelas classes: i) MKnowledgeDomain e MConcept: as classes MKnowledgeDomain e MConcept representam o conjunto de conceitos relevantes do domínio do conhecimento. ii) Screen: a classe Screen é composta pelos os demais elementos do conteúdo educacional (texto, imagem, áudio, atividade avaliativa) e representa uma tela do aplicativo. iii) Texto, Image, Audio, Video: essas classes representam o formato do conteúdo educacional que será atribuído a cada Screen.

Cabe destacar que o Metamodelo Mobile não modela uma plataforma real de destino (Android, IOS, Windows Mobile). Essa abordagem foi adotada por fornecer versalidade à equipe de desenvolvimento, que a partir de um modelo genérico pode obter modelos específicos para plataformas existentes no mercado.

\section{Metamodelo XMLAndroid}

Nesse estudo, o conteúdo educacional tem como alvo a plataforma Android. Logo, para obter um modelo real da plataforma Android foi proposto o Metamodelo XMLAndroid. O Metamodelo XMLAndroid representa o padrão XML Android utilizado para implementar layput de telas de aplicativos Android. No processo de transformação do Modelo Mobile em Modelo XMLAndroid as seguintes classe são instanciadas: i) Activity: no padrão XML Android cada tela é representada por uma Activity, assim, essa classe tem o objetivo agrupar elementos que compõe a tela do aplicativo. ii) Layout: a classe Layout tem o objetivo de definir a forma de organização do elementos de uma Activity. A classe Layout é uma generalização das classes FrameLayout, LinearLayout, GridLayput e TableLayput. iii) Os elementos presentes nas Activity são representados pelas classes TextView, ImageView, Button, EditText, entre outros. 
VII Congresso Brasileiro de Informática na Educação (CBIE 2018)

Anais do XXIX Simpósio Brasileiro de Informática na Educação (SBIE 2018)

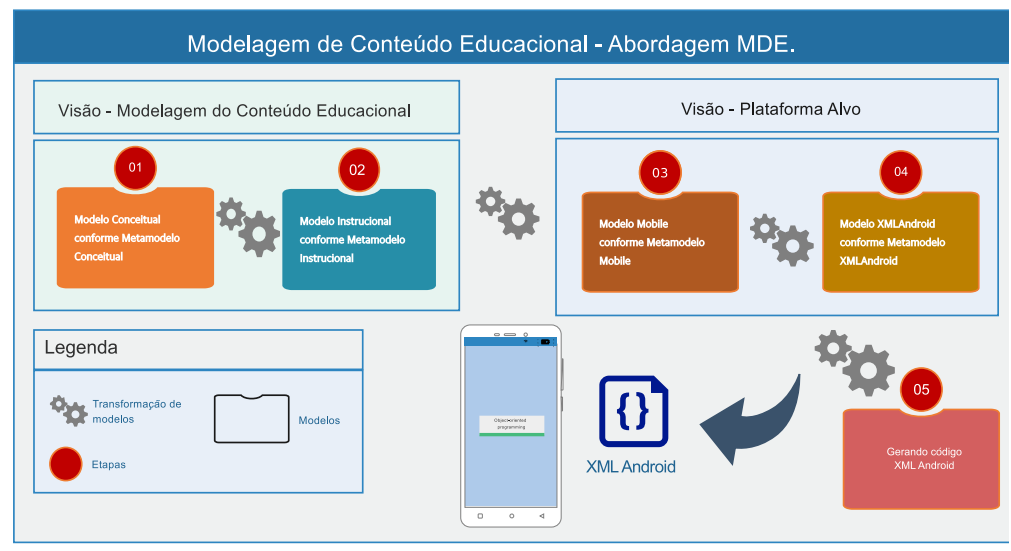

Figura 1. Abordagem MDE aplicada na modelagem de conteúdo educacional móvel.

Em síntese, a modelagem de conteúdo educacional proposta estabelece uma divisão clara de responsabilidade na equipe de produção do conteúdo. Professores/autores mantém foco nas questões didáticas do conteúdo construindo o Modelo Conceitual e Instrucional, enquanto que a equipe de desenvolvimento concentra esforços em construir modelos da plataforma de destino.

\section{Aplicando a abordagem de modelagem de conteúdo educacional móvel}

A modelagem de conteúdo educacional móvel foi aplicada ao domínio da Programação Orientada a Objetos (POO). Um XML Android com os conceitos mais relevantes de POO foi obtido a partir de instâncias dos modelos propostos na modelagem de conteúdo educacional móvel. $\mathrm{O} \mathrm{EMF}^{1}$ foi utilizado para implementação dos metamodelos, regras de transformação e instanciação dos modelos. Como ilustra a Figura 1, a modelagem do conteúdo educacional móvel segue as seguintes etapas:

1. Instanciar o Modelo Conceitual para POO. A Figura 2(a) ilustra um fragmento do Modelo Conceitual instanciado para o domínio da POO. Foram modelados conceitos de POO como Class, Operation, Attribute, Inheritance entre outros. Além de conceitos, foram atribuídas propriedades que representam os relacionamentos como o typeOf existentes entre os conceitos Single Inheritance e Inheritance.

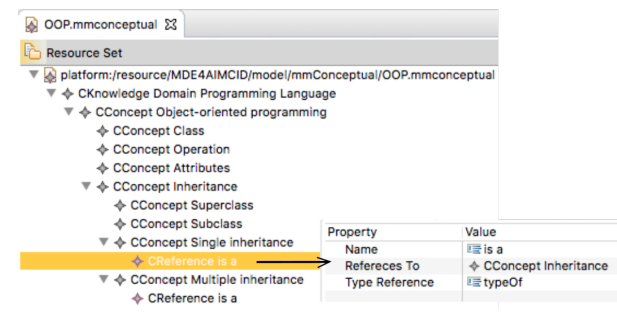

(a) Modelo Conceitual para POO (fragmento)

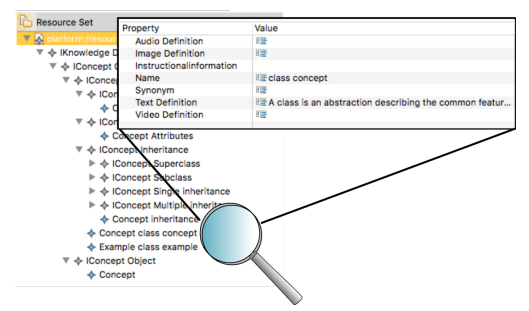

(b) Modelo Instrucional para o domínio POO (fragmento)

Figura 2. Modelagem do conteúdo educacional do domínio de POO

\footnotetext{
${ }^{1}$ Eclipse Modeling Framework, https://projects.eclipse.org/projects/modeling.emf.emf
} 
VII Congresso Brasileiro de Informática na Educação (CBIE 2018)

Anais do XXIX Simpósio Brasileiro de Informática na Educação (SBIE 2018)

2. Instanciar o Modelo Instrucional para POO. Com o Modelo Conceitual definido, regras de transformações foram aplicadas a ele obtendo-se o Modelo Instrucional. Como pode ser visto na Figura 2(b), o Modelo Instrucional obtido foi complementado com o item de informação (Example e Concept). A classe (Concept) possui o atribuído (textDefinition), que contém a descrição do conceito de Classe em POO.

3. Instanciar o Modelo Mobile. Nesta etapa, regras de transformação são aplicadas ao Modelo Instrucional obtendo-se o Modelo Mobile. O Modelo Mobile contém uma representação genérica do conteúdo que será apresentado nas telas do aplicativo móvel. A Figura 3(a) apresenta o Modelo Mobile do domínio POO instanciado. Observe que o conceito de Classe possui uma Screen que será composta por um Text e uma Imagem. Na etapa seguinte, o modelo real de plataforma será obtido(plataforma Android). O Modelo Mobile instanciado nesta etapa pode ser transformado em modelos das paltaformas Android, IOS ou Windows Mobile.

4. Instanciar o Modelo XMLAndroid. O Modelo XMLAndroid contém exatamente os elementos que são fornecidos pela plataforma Android para construção de layout de aplicativos. Portanto, as telas modeladas no Modelo Mobile serão transformadas em elementos do Modelo XMLAndroid. Como ilustrado na Figura 3(b), o conceito de Classe será exibido em uma Activity contendo um TextView e um ImageView. Para cada conceito representado no Modelo Mobile foi criado uma Activity com a composição de elementos Views adequado ao conteúdo.

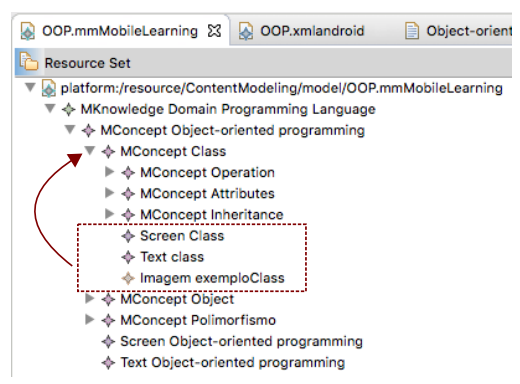

(a) Modelo Mobile para o domínio POO (fragmento)

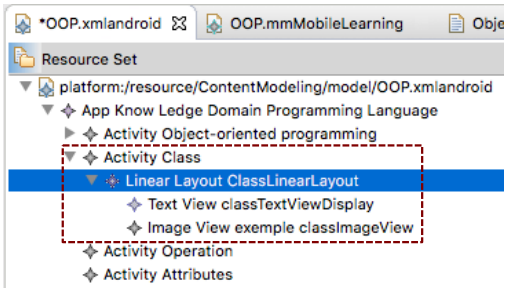

(b) Modelo XMLAndroid para o domínio POO (fragmento)

Figura 3. Modelagem da plataforma tecnológica.

5. Gerar código XML Android. Por fim, regras de transformação foram aplicadas ao Modelo XMLAndroid gerando o conteúdo educacional móvel para plataforma Android, conforme pode ser vizualizado nas Figuras 4(a)(regras de tranformação) e 4(b)(XML Android gerado).

\section{Conclusões e Trabalhos Futuros}

A utilização de tecnologias móveis no contexto da educação está modificando a maneira como professores e aprendizes produzem e consomem conteúdo educacional. Atualmente, cursos completos são oferecidos por meio de plataformas móveis. Isso gera a necessidade de um formalismo na produção de conteúdo educacional. Este artigo apresentou a proposta de modelagem de conteúdo educacional móvel baseada na abordagem AIM-CID e na aplicação da MDE para a geração semi-automática de conteúdo educacional móvel. 
VII Congresso Brasileiro de Informática na Educação (CBIE 2018)

Anais do XXIX Simpósio Brasileiro de Informática na Educação (SBIE 2018)

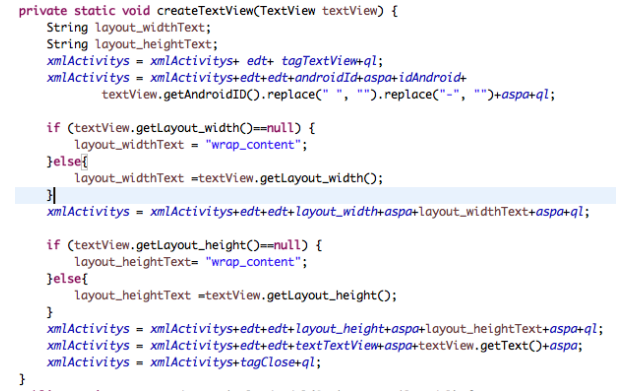

(a) Regra de transformação de elemento TextView.

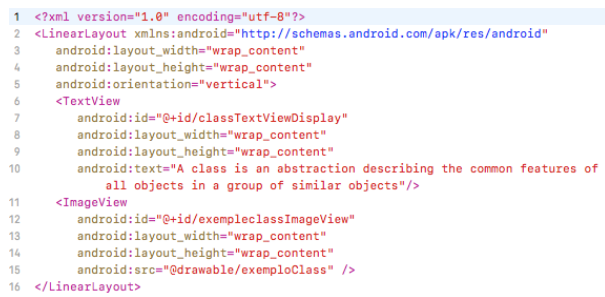

(b) Activity gerada para o conceito de Classe do domínio POO

Figura 4. Conteúdo digital gerado por transformação dos modelos conceitual e instrucional

A modelagem de conteúdo educacional apresentada propõe duas visões. Uma visão de modelagem de conteúdo educacional desconsiderando aspectos tecnológicos da plataforma alvo; e outra visão de modelagem de conteúdo educacional centrada em aspectos tecnológicos da modalidade de ensino e aprendizagem. Assim, o professor/autor pode concentrar esforços somente em especificar as características do conteúdo didático a ser apresentado ao aprendiz, enquanto que a equipe de desenvolvimento preocupa-se nos aspectos tecnológicos da plataforma de consumo do conteúdo didático. Além disso, essas duas visões permitem gerar conteúdo educacional para plataformas tecnológicas distintas (móvel, web, tradicional). Essa flexibilidade é conseguida com uso da abordagem de desenvolvimento de software MDE.

Como trabalhos futuros, a serem realizados de curto a médio prazo, destacamse: i) propor um metamodelo para o Modelo Didático da AIM-CID; ii) desenvolver uma ferramenta que auxilie o trabalho de produção de conteúdo educacional para professor/autor; iii) identificar características tecnológicas e propor metamodelos para gerar conteúdo educacional para plataformas IOS e Web. iv) validar com professores e aprendizes o uso da modelagem de conteúdo educacional móvel.

\section{Agradecimentos}

Os autores agradecem o apoio da Coordenação de Aperfeiçoamento de Pessoal de Nível Superior - Brasil (CAPES) - Código de Financiamento 001, FAPESP e CNPq.

\section{Referências}

Apple (2018). Human interface guidelines. Available at http://developer.apple.com/ios. Acessed at 05/04/2018.

Association, G. (2017). The mobile economy. GSM Association. Available at https://www.gsma.com/. Acessed at 05/04/2018.

Barbosa, E. F., Borges, V. A., and Maldonado, J. C. (2013). Introdução à Modelagem de Conteúdos Educacionais. Jornada de Atualização em Informática na Educação, 1(1):82-120.

Barbosa, E. F. and Maldonado, J. C. (2011). Ima-cid: an integrated modeling approach for developing educational modules. Journal of the Brazilian Computer Society, 17(4):207-239. 
VII Congresso Brasileiro de Informática na Educação (CBIE 2018)

Anais do XXIX Simpósio Brasileiro de Informática na Educação (SBIE 2018)

Biard, N., Cojean, S., and Jamet, E. (2017). Effects of segmentation and pacing on procedural learning by video. Computers in Human Behavior.

Chittaro, L. (2010). Distinctive aspects of mobile interaction and their implications for the design of multimodal interfaces. Journal on Multimodal User Interfaces, 3(3):157165 .

Conde, A., Larrañaga, M., Calvo, I., Elorriaga, J. A., and Arruarte, A. (2012). Automating the authoring of learning material in computer engineering education. In Frontiers in Education Conference (FIE), 2012, pages 1-6. IEEE.

DE SOUZA, M. de Fátima C.; DE CASTRO FILHO, J. A. A. R. M. (2012). Customização Guiada: uma estratégia Orientada a Modelos para a Produção de Objetos de Aprendizagem. Anais do Simpósio Brasileiro de Informática na Educação, 23(1).

Diwanji, P., Simon, B. P., Marki, M., Korkut, S., and Dornberger, R. (2014). Success factors of online learning videos. In 2014 International Conference on Interactive Mobile Communication Technologies and Learning (IMCL2014), pages 125-132.

EMF (2018). Eclipse modeling framework project. Eclipse Modeling Framework Project (EMF). Available at http://www.eclipse.org/modeling/emf/. Acessed at 05/01/2018.

Google (2018). Introducing material design. Developer Android. Available at https://developer.android.com/design/. Acessed at 05/04/2018.

IBGE (2018). Pesquisa nacional por amostra de domicílios - 2014: síntese de indicadores. Instituto Brasileiro de Geografia e Estatística - IBGE. Available at http://biblioteca.ibge.gov.br/visualizacao/livros/liv94935.pdf. Acessed 01/04/2018.

Joorabchi, M. E., Mesbah, A., and Kruchten, P. (2013). Real challenges in mobile app development. In 2013 ACM / IEEE International Symposium on Empirical Software Engineering and Measurement, pages 15-24.

Mayer, R. (2017). Using multimedia for e-learning. Journal of Computer Assisted Learning, 33(5):403-423.

Merrill, M. D. (1983). Component display theory. Instructional-design theories and models: An overview of their current status, 1:282-333.

Minović, M., Milovanović, M., Starcevic, D., and Jovanović, M. (2009). Knowledge modeling for educational games. In World Summit on Knowledge Society, pages 156165. Springer.

Navarro, C. X., Molina, A. I., Redondo, M. A., and Juárez-Ramírez, R. (2016). Framework to evaluate m-learning systems: A technological and pedagogical approach. IEEE Revista Iberoamericana de Tecnologias del Aprendizaje, 11(1):33-40.

Prieto, J. C. S., Migueláñez, S. O., and García-Peñalvo, F. J. (2013). Understanding mobile learning: devices, pedagogical implications and research lines. Education in the Knowledge Society, 15(1):20.

Whittle, J., Hutchinson, J., and Rouncefield, M. (2014). The state of practice in modeldriven engineering. IEEE Software, 31(3):79-85. 